\title{
A EPIDEMIOLOGIA DA TUBERCULOSE HUMANA NO MUNDO
}

\author{
Cícero Pereira Batista ${ }^{1}$
}

\section{RESUMO}

A tuberculose, apesar de ter sido descoberta há muito tempo e contar com tratamento eficaz tanto para a prevenção quanto para a cura, ainda é um problema de saúde pública em muitos países. Posto isso, o presente artigo tem a meta de descrever como a tuberculose se manifesta na população mundial a partir de dados fornecidos pelas organizações de saúde do mundo como um todo. Por meio de um levantamento bibliográfico, pretende-se dar visibilidade à comunidade acadêmica e interessados pelo tema de modo geral para essa doença que pode ser letal quando não tratada.

Palavras-chave: Tuberculose; Prevenção; Saúde.

\begin{abstract}
Tuberculosis, although it has been discovered for a long time and has effective treatment for both prevention and cure, is still a public health problem in many countries. Having said that, this article aims to describe how tuberculosis manifests itself in the world population from data provided by health organizations around the world as a whole. Through a bibliographic survey, it is intended to give visibility to the academic community and interested in the theme in general for this disease that can be lethal when untreated.
\end{abstract}

Keywords: Tuberculosis; Prevention; Health.

\section{RESUMEN}

La tuberculosis, aunque se ha descubierto durante mucho tiempo y tiene un tratamiento eficaz tanto para la prevención como para la curación, sigue siendo un problema de salud pública en muchos países. Dicho esto, este artículo tiene como objetivo describir cómo la tuberculosis se manifiesta en la población mundial a partir de datos proporcionados por organizaciones de salud de todo el mundo en su conjunto. A través de una encuesta bibliográfica, se pretende dar visibilidad a la comunidad académica e interesado en el tema en general para esta enfermedad que puede ser letal cuando no se trata.

Palabras clave: Tuberculosis; Prevención; Salud.

\footnotetext{
${ }^{1}$ Especialização em Psiquiatria em andamento, Centro Brasileiro de Pós-Graduação (Cenbrap). Pós Graduando em Saúde Mental pela Faculdades Integradas (AVM). Possui graduação em Medicina - Faculdades Integradas da União Educacional do Planalto Central (2014). Atualmente é médico clínico geral - Secretaria de Saúde Planaltina de Goiás e médico clínico geral Secretaria de Saúde Águas Lindas. Tem experiência na área de Medicina, com ênfase em Medicina Familiar.
} 


\section{INTRODUÇÃO}

A temática tuberculose (TB) surgiu de uma das muitas inquietações que persiste dentre os profissionais de saúde devido, especialmente, a esta ser uma doença considerada antiga, prevenível, possuir vacina e, ainda assim, ser considerada um problema de saúde pública em muitos países.

Além das publicações científicas, a mídia em geral, anualmente, divulga dados alarmantes das doenças que são a principais causas de morte no mundo. A fim de exemplificação, estão arroladas em vários estudos científicos e publicações jornalísticas as dez principais causas de morte no mundo por ordem de letalidade, como a Cardiopatia isquêmica; o Acidente vascular cerebral (AVC); a Doença pulmonar obstrutiva crônica; as Infecções das vias respiratórias inferiores; o Alzheimer e outras demências; o Câncer de pulmão, traqueia e brônquios; o Diabetes mellitus; os Acidentes de trânsito; as Doenças diarreicas; e a Tuberculose (BRASIL, OPAS 2020 p. 1).

Ainda, de acordo com OMS (2018), no ano de 2016, as principais causas de morte no mundo têm entre elas a tuberculose como causadora, apesar desta doença de melhorado seu status no ranking, já que em 2000 ocupava a sexta colocação e em 2018 a décima, levando a óbito mais de 1,5 milhões de seres humanos.

No mundo, várias são as doenças que são classificadas como um grave problema de saúde pública, já que milhões de pessoas ainda adoecem e morrem devido as suas complicações. Na Assembleia Mundial de Saúde, na Organização Mundial da Saúde (OMS), em 2014, foi aprovada a nova estratégia global para enfrentamento da tuberculose, com a visão de um mundo livre da tuberculose até 2035. Momento este em que o Brasil teve o papel de destaque ao ser o principal proponente da estratégia e, principalmente, por sua experiência com o Sistema Único de Saúde (SUS) e com a Rede Brasileira de Pesquisas em Tuberculose (Rede-TB).

De acordo com a Coordenação-Geral do Programa Nacional de Controle da Tuberculose Departamento de Vigilância de Doenças Transmissíveis Secretaria de Vigilância em Saúde/Ministério da Saúde (2017, p. 2): 
O Brasil é um dos países com maior número de casos no mundo e, desde 2003, a doença é considerada como prioritária na agenda política do Ministério da Saúde (MS). Embora seja uma doença com diagnóstico e tratamento realizados de forma universal e gratuita pelo Sistema Único de Saúde, ainda temos barreiras no acesso e acontecem 69 mil casos novos e 4.500 óbitos a cada ano, tendo como causa básica a tuberculose (BRASIL, 2017, p. 2).

Pode-se perceber que, mesmo não sendo considerada a doença que mais mata no mundo, a tuberculose possui alta taxa de letalidade mesmo sendo evitável por meio de administração de vacina e, ainda, tratável com medicamentos específicos.

Em se tratando de uma doença como a tuberculose, que muitos estudos já foram e continuam sendo desenvolvidos, a patogenia, a prevenção por meio de vacina, as formas de tratamento tornam-se um grande desafio, sendo imperioso estudar a epidemiologia da doença, considerando o grande número de vítimas fatais que ainda ocorrem.

Tendo em vista esse cenário problemático, o presente artigo tem a meta de analisar os aspectos históricos, políticos e sociais da tuberculose no mundo a fim de dar visibilidade à temática e evitar o contágio e, consequentemente, o elevado número de óbitos no mundo.

Para tal, este estudo divide-se em Questões iniciais sobre a tuberculose do mundo, com o aprofundamento sobre questões mais comuns e históricas sobre a doença em si; Características da doença, com o perfilamento da doença com base na literatura médica mundial; $A$ epidemiologia da tuberculose humana no Mundo, que busca, mais diretamente, atender ao objetivo geral deste artigos, considerado descrever como a tuberculose se manifesta na população mundial a partir de dados fornecidos pelas organizações de saúde do mundo como um todo.

Em acréscimo, têm-se a seção destinada à Metodologia, Conclusões e Referências Bibliográficas utilizadas.

\section{QUESTÕES INICIAIS SOBRE A TUBERCULOSE}

Entende-se que qualquer doença classificada como um problema de saúde pública no mundo ou no Brasil por si só já se justifica como sendo um importante objeto de pesquisa científica. Desse modo, segundo dados levantados pela 
Organização Mundial da Saúde (OMS), em 2017, de 10 milhões de casos de tuberculose registrados no mundo, 1,6 milhões foram fatais, superando até mesmo o número de mortes por AIDS. Nesse mesmo ano, foram registrados em torno de 4,5 mil mortes por tuberculose no Brasil - muitas delas verificadas em pacientes portadores do HIV - número que fez com que a OMS colocasse 0 país na lista de nações prioritárias para o combate à doença.

Para iniciar esta breve investigação sobre a história da tuberculose no mundo, torna-se necessário responder algumas questões que, no cotidiano profissional, sempre vêm à tona. Assim, acredita-se que estas, provavelmente, são feitas no intuito de melhor compreender a doença face as muitas especulações populares que são comentadas no dia a dia das pessoas e, muitas vezes, de forma inadequada e pejorativa.

O primeiro questionamento reside no fato de a população em geral conhecer sua real origem. De acordo com estudos já amplamente divulgados, a tuberculose surgiu na África há 70.000 anos e possui uma trajetória evolutiva próxima a dos seres humanos. Essa descoberta foi possível por meio de estudos internacionais realizados a partir da análise de 259 amostras da bactéria Mycobacterium tuberculosis, causadora da doença.

No encalço desse questionamento, há também a relevância de se apresentar quando essa doença passou a ser curável e evitável. Em 24 de março de 1882, o doutor Robert Koch, médico alemão, descreveu o bacilo causador da tuberculose, Mycobacterium tuberculosis já citado aqui. Este médico, pela sua descoberta, em 1905 recebeu o Prêmio Nobel de Medicina, apresentando, ainda, em 1890, um remédio denominado tuberculina para o tratamento da tuberculose, que, se administrado corretamente, cura a doença em seis meses.

Já há vacina, conhecida como BCG, foi criada pelos pesquisadores Albert Calmette e Camille Guerin a partir de uma bactéria responsável por desencadear mastite tuberculosa bovina, a Mycobacterium bovis. Sua primeira utilização foi feita em uma criança recém-nascida de mãe que apresentava tuberculose em 1921 (SANTOS, 2020 p. 1).

Outra questão comum entre os indivíduos em geral e interessados pelo assunto é sobre as formas de contágio. A transmissão, geralmente, se dá por meio do ar, a tosse, o cuspir, o espirro e a fala de uma pessoa que está infectada 
lança os bacilos no ambiente e quem convive próximo ao infectado aspira esses bacilos e pode, também, se tornar infectado.

No Brasil, diferentemente de outras nações, no Brasil, há tratamento no Sistema Único de Saúde e é totalmente gratuito. Esta iniciativa deixou o país com números mais promissores de controle da doença nos últimos anos, mas ainda longe do ideal.

\section{CARACTERÍSTICAS DA DOENÇA}

Após ter apresentado algumas questões iniciais sobre a tuberculose consideradas as principais dúvidas nas consultas clínicas, agora serão apresentadas as características gerais da doença e, em especial, seus sinais, sintomas, formas de transmissão, diagnóstico, prevenção e tratamento da tuberculose humana.

Entende-se que um profissional de saúde experiente, provavelmente, não terá dificuldade de identificar alguns prováveis sinais e sintomas da tuberculose humana, em especial, conhecendo um pouco mais o nível socioeconômico do paciente, suas condições de moradia e hábitos da vida diária, pois estas informações, também, são importantes para solicitar exames específicos para o diagnóstico da tuberculose.

Posto isso, os principais sinais e sintomas de um paciente possivelmente infectado são a tosse, com ou sem secreção, que pode ser espessa ou até com a presença de sangue, falta de apetite, perda de peso, rouquidão, cansaço excessivo, falta de ar, febre baixa, mais comum à tarde, e sudorese noturna.

\footnotetext{
Em 1993 a OMS declarou a Tuberculose (TB) uma emergência mundial. Em agosto de 2005 o 55을 Comité Regional da OMS, reunido em Maputo, declarou a TB uma emergência em África. DOTS é o nome da estratégia adoptada pela OMS para o controlo da Tuberculose a nível mundial e que se encontra incluída no "Estratégia Stop TB-2006-2015". A estratégia DOTS engloba 5 componentes: 1. Compromisso político mantido de forma sustentável. 2. Despiste dos casos através da bacteriologia, com garantia de controlo de qualidade. 3. Tratamento da TB com regimes de curta duração estandardizados, com supervisão e apoio aos doentes. 4. Sistema eficaz, sem roturas no fornecimento e gestão de medicamentos anti-TB. 5. Sistema de registo, notificação e avaliação dos casos de TB a nível nacional e medição do impacto (PERDIGÃO, 2008, p. 3).
}

Causada pela bactéria Mycobacterium tuberculosis, também conhecida como bacilo de Koch, a tuberculose pulmonar é transmitida pelo ar, por meio de 
gotículas provenientes de tosse ou espirro de uma pessoa infectada, uma pessoa sadia que ao respirar o ar contaminado inala as microbactérias, que se instalam nos pulmões. Pode, também, ocorrer em outros órgãos do corpo, como ossos, rins e meninges (membranas que envolvem o cérebro).

Quanto ao diagnóstico, a tuberculose humana é classificada em dois tipos principais, a mais frequente a tuberculose pulmonar, e a tuberculose miliar ou extrapulmonar, sendo esta originada do primeiro tipo, quando não adequadamente tratada. A literatura ainda subdivide em outras formas que serão detalhadas aqui.

Desse modo, a tuberculose pulmonar surge no paciente quando a fixação do bacilo fixa nos alvéolos pulmonares. É a forma mais comum da doença e inicia-se com a fixação da Mycobacterium tuberculosis nos alvéolos pulmonares, que após, a penetração do hospedeiro no individuo, por meio das vias aéreas superiores (nariz e garganta), alcança os pulmões e inicia seu processo de multiplicação, gerando a necrose dos tecidos. A tuberculose pulmonar se divide em primária, quando afeta indivíduos que não tenham sido previamente expostos ao bacilo (agente causador), sendo mais comum na infância, fase em que o sistema imunológico ainda não se encontra totalmente desenvolvido; e secundária, surgindo tanto devido a uma nova infecção quanto em decorrência da reativação de bacilos que tenham se mantido no organismo.

Outro tipo de tuberculose chama-se ganglionar, mais comum em crianças e portadores do HIV soropositivos. Pacientes com esta forma da doença podem apresentar dor e inchaço dos gânglios, que são glândulas linfáticas localizadas no pescoço. Ainda, a tuberculose extrapulmonar é a forma mais comum em portadores do HIV soronegativos. Além dos sintomas mais gerais, geralmente há dor na região torácica.

Ampliando seu campo de atuação, a meningite tuberculosa caracteriza-se pela infecção das membranas que envolvem o sistema nervoso central. Outro tipo, mais raro, é a tuberculose intestinal que pode ser transmitido por meio da ingestão de leite não pasteurizado e contaminado pelo Mycobacterium bovis, ou resultar de complicações da tuberculose pulmonar. Os sintomas mais característicos, neste caso, são febre e diarreia.

Há, inclusive, a tuberculose óssea que afeta as articulações e ossos, sobretudo os da coluna (vértebras) e os longos, como os das coxas, causando 
dores nessas regiões. É mais comum em crianças e adultos a partir dos 40 anos. Por fim, a literatura médica elenca a tuberculose cutânea, sendo a forma mais rara de tuberculose extrapulmonar, com apenas $1,4 \%$ de casos registrados. Caracteriza-se pelo surgimento de nódulos avermelhados na pele, e em adultos, pode ser causada pela vacina BCG, caso o paciente lesione a área de aplicação da vacina, em casos recentes da vacinação. Nesses quadros, a tuberculose cutânea provoca erupções na pele que se assemelham a feridas e lesões.

É importante lembrar que as tuberculoses não pulmonares, como a cutânea, não são contagiosas e o diagnóstico pode ser feito de forma mais precisa por meio de radiografias de tórax, por exemplo, como pode ser observado a seguir na radiografia do peito de uma pessoa com tuberculose avançada. As setas brancas assinalam a infeção em ambos os pulmões. As setas pretas assinalam a formação de uma cavidade.

Figura 1. Radiografia do Tórax

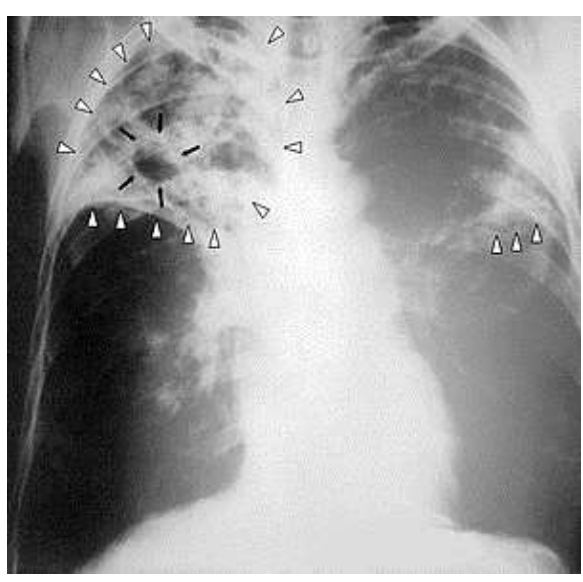

Fonte: https://pt.wikipedia.org/wiki/Tuberculose (2020).

Outros exames que podem identificar a presença da doença são os exames microscópicos, as culturas de fluidos corporais, análises do sangue e a chamada prova da tuberculina, conhecida, ainda, como Teste Tuberculínico ou Reação de Mantoux que consiste na inoculação intradérmica de um derivado protéico do M. tuberculosis (PPD) para medir a resposta imune celular a estes antígenos. Nos indivíduos já sensibilizados, ocorre uma reação inflamatória (DUARTE, 2009).

Quanto as medidas de prevenção e de tratamento da tuberculose humana, tem-se o rastreio de grupos de risco, detecção e tratamento atempados dos 
casos e vacinação com o bacilo Calmette-Guérin (vacina BCG). Os grupos de risco incluem as pessoas que partilham casa ou local de trabalho com pessoas com tuberculose ativa. O tratamento consiste na administração de vários antibióticos durante um longo período de tempo. A resistência antibiótica é um problema crescente, estando a aumentar o número de casos de tuberculose multirresistente (MDR-TB) e a tuberculose extensivamente resistente (XDR-TB).

\section{A EPIDEMIOLOGIA DA TUBERCULOSE HUMANA NO MUNDO}

Como já foi dito anteriormente, é uma doença que acompanha os seres humanos há mais de setenta mil anos e que é um grande desafio à ciência. Um estudo publicado na revista Nature Genetics, sobre a tuberculose, liderado por Sébastien Gagneux, do Instituto de Saúde Pública e Tropical Suíço, pesquisou 259 amostras da bactéria causadora da enfermidade, a Mycobacterium tuberculosis, colhidas em várias partes do mundo e a comparação das árvores evolutivas do homem e do bacilo apresentou muitas semelhanças, com a indicação, inclusive, de que ambos surgiram na África e migraram juntos para o restante do planeta.

\footnotetext{
O desenvolvimento da agricultura, as mudanças climáticas produzidas pelo recuo das geleiras e a elevação do nível dos mares durante o Período Neolítico ofereceram ao homem as condições para a migração. A combinação mostrou-se cenário perfeito também para a disseminação da tuberculose. "O fato de as pessoas terem começado a morar em vilas e o crescimento populacional devem ter potencializado a virulência da doença, já que a transmissão entre humanos passou a ocorrer mais facilmente", sugere Sébastien Gagneux. Entre os séculos 17 e 19, a tuberculose exterminou 20\% da população mundial, e ainda hoje tem uma alta taxa de mortalidade em países em desenvolvimento. Dados do Ministério da Saúde mostram que em 2012, 71.230 casos foram registrados no Brasil. Se não for tratada, a doença mata $50 \%$ dos infectados (CORREIO BRAZILIENSE, 2013 p. 1).
}

Segundo mesmo autor, estima-se que cerca de um terço da população mundial esteja infetada com tuberculose latente. Todos os anos, ocorrem novos casos de infeção em cerca de $1 \%$ da população. Em 2016, ocorreram em todo o mundo mais de 10 milhões de casos de tuberculose ativa, tendo sido a causa de 
cerca de 1,3 milhões de mortes. Isto faz com que a tuberculose seja a principal motivo de letalidade por doenças infeciosas. Mais de $95 \%$ destas mortes ocorreram em países em desenvolvimento, em especial, na Índia, China, Indonésia, Paquistão e Filipinas.

Desde o ano 2000, que o número de novos casos em cada ano tem vindo a diminuir. Em muitos países africanos e asiáticos cerca de $80 \%$ da população apresenta prova de tuberculina positiva, enquanto nos Estados Unidos este valor é de apenas 5-10\% da população. A doença está presente nos seres humanos desde a Antiguidade.

De acordo com a OMS (2016), citada na obra Brasil livre de tuberculose (2017), a OMS preparou três listas com trinta países em cada uma, de países prioritários no combate a três tipos de tuberculose, como tem país que está em mais de uma lista, a mesma alcança o número de 48 países prioritários no combate à doença. O Brasil faz parte de duas listas ocupando a 20aㅡ posição quanto à carga da doença (TB) e a 19 $9^{a}$ no que se refere à coinfecção tuberculose-HIV (TB-HIV):

Quadro 1. Classificação de países prioritários para o período de 2016 a 2020

\begin{tabular}{|l|l|l|l|}
\hline \multicolumn{1}{|c|}{ PAÍSES LISTADOS } & \multicolumn{1}{c|}{ Tuberculose } & $\begin{array}{c}\text { Tuberculose } \\
\text { associada ao HIV }\end{array}$ & $\begin{array}{l}\text { Tuberculose } \\
\text { multidrogarresistente }\end{array}$ \\
\hline Camboja & $\mathrm{X}$ & & \\
\hline Serra Leoa & $\mathrm{X}$ & & \\
\hline África do Sul & $\mathrm{X}$ & & \\
\hline Angola & $\mathrm{X}$ & & \\
\hline China & $\mathrm{X}$ & & \\
\hline Etiópia & $\mathrm{X}$ & & \\
\hline Índia & $\mathrm{X}$ & & \\
\hline Indonésia & $\mathrm{X}$ & & \\
\hline Moçambique & $\mathrm{X}$ & & \\
\hline Myanmar & $\mathrm{X}$ & & \\
\hline Nigéria & $\mathrm{X}$ & & \\
\hline Papua Nova Guiné & $\mathrm{X}$ & & \\
\hline Quênia & $\mathrm{X}$ & & \\
\hline Rep. Democrática do & $\mathrm{X}$ & & \\
Congo & $\mathrm{X}$ & & $\mathrm{X}$ \\
\hline Tailândia & $\mathrm{X}$ & & $\mathrm{X}$ \\
\hline Zimbábue & $\mathrm{X}$ & & $\mathrm{X}$ \\
\hline Bangladesh & $\mathrm{X}$ & & $\mathrm{X}$ \\
\hline Coreia do Norte & $\mathrm{X}$ & & \\
\hline Federação Russa & $\mathrm{X}$ & & \\
\hline Filipinas & $\mathrm{X}$ & $\mathrm{X}$ & \\
\hline Paquistão & $\mathrm{X}$ & & \\
\hline Vietnã & $\mathrm{X}$ & & \\
\hline Brasil & & & \\
\hline
\end{tabular}




\begin{tabular}{|c|c|c|c|}
\hline $\begin{array}{l}\text { República Centro } \\
\text { Africana }\end{array}$ & $\mathrm{X}$ & $X$ & \\
\hline Congo & $X$ & $X$ & \\
\hline Lesoto & $X$ & $X$ & \\
\hline Libéria & $X$ & $X$ & \\
\hline Namíbia & $\mathrm{X}$ & $\mathrm{X}$ & \\
\hline Tanzânia & $X$ & $X$ & \\
\hline Botsuana & & $X$ & \\
\hline Cameroun & & $X$ & \\
\hline Chade & & $X$ & \\
\hline Gana & & $X$ & \\
\hline Guiné Bissau & & $\mathrm{X}$ & \\
\hline Maláui & & $\mathrm{X}$ & \\
\hline Suazilândia & & $\mathrm{X}$ & \\
\hline Uganda & & $\mathrm{X}$ & \\
\hline Azerbaijão & & & $\mathrm{X}$ \\
\hline Bielorrússia & & & $\mathrm{X}$ \\
\hline Cazaquistão & & & $X$ \\
\hline Quirguistão & & & $X$ \\
\hline Peru & & & $\mathrm{X}$ \\
\hline Moldávia & & & $X$ \\
\hline Somália & & & $X$ \\
\hline Tajiquistão & & & $x$ \\
\hline Ucrânia & & & $x$ \\
\hline
\end{tabular}

Fonte: Adaptado de Brasil livre de tuberculose (2017, p. 9).

De acordo com Teixeira (2003), no editorial do Boletim de pneumologia sanitária, em observação ao mapa-múndi, para expressar a magnitude da tuberculose no mundo, observa-se que os países e continentes mais afetados estão diretamente relacionados à representação geográfica da miséria, do subdesenvolvimento e da desorganização social no mundo. Destaca-se, por exemplo, que os países africanos são os mais atingidos, com taxas de incidência superiores a 300 casos/100.000 habitantes, como também o Sudeste da Ásia, da América Latina, da Europa, da América do Norte - que, neste caso, as taxas são inferiores a 10 casos/100.000.

Ainda, o mesmo autor diz que dos 22 países que concentram a maior carga de tuberculose - $80 \%$ do total de casos do mundo - 9 estão na África, 5 no Sudeste da Ásia, 4 no Pacífico-Ocidental, 2 no Mediterrâneo-Oriental, 1 na Europa e 1 na América do Sul (Brasil). Entretanto, não se pode deixar de salientar que mesmo em países desenvolvidos, industrializados, há também, em seus grandes centros urbanos, em especial nas periferias destes países ou grandes cidades, índices de tuberculose comparáveis à países africanos. 
O artigo "Tuberculose, Vila Rosário e a Cadeia da Miséria" que aparece neste número do Boletim, de autoria de Cláudio Costa Neto - um professor universitário de Química, idealista, para quem o social é a grande via que leva à erradicação das mazelas da sociedade - defende, com base em experiência desenvolvida em uma área de miséria da periferia do Rio de Janeiro que, para a eliminação de problemas como o da tuberculose, se impõe uma ação holística, pactuada, de todas as forças da comunidade. Este caminho passa pela conquista, para todos, da educação, da assistência à saúde, da segurança, do emprego, dos direitos de cidadania, da democracia, enfim. $\mathrm{Na}$ medida em que avançamos, na observação do planeta, sob a ótica dos preceitos da globalização, mas nos damos conta de quão forte é o papel da interdependência dos países no agravamento da problemática de saúde. Fica claro, neste particular, que cada dia é mais difícil alimentar políticas isolacionistas voltadas para a construção de ilhas de salubridade que coincidam com as fronteiras da riqueza (TEIXEIRA, 2003 p. 1).

Cada vez se torna mais claro que ações em saúde pública visando, por exemplo, controle e erradicação de doenças, deva levar em conta a visão global, pois entende-se que os indivíduos são um só mundo e as doenças transmissíveis, como a tuberculose, fazem parte de uma extensa lista de doenças que sofrem influências migratórias, dos deslocamentos das pessoas entre países e continentes, da economia global, da massificação do turismo no mundo, dentre muitos outros fatores.

Nesta direção, em 2015, foram fixados pela Organização das Nações Unidas os 8 Objetivos de Desenvolvimento do Milênio (ODM) e os 17 Objetivos de Desenvolvimento Sustentável, além de 169 metas a serem alcançadas até 2030, das quais várias são ligadas a saúde da população e do planeta.

Figura 2. Objetivos de Desenvolvimento do Milênio (2000)

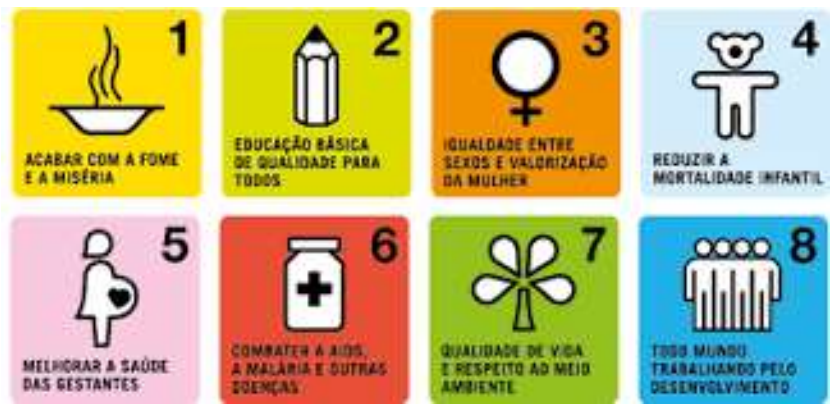

Fonte: OPAS/OMS Brasil 2020.

Segundo a OPAS/OMS (2020, p. 1), os ODS são integrados e indivisíveis e equilibram as três dimensões do desenvolvimento sustentável: econômica, social e ambiental. Eles estimularão a ação para os próximos anos em áreas de importância crucial para a humanidade e para o planeta e abrange um conjunto 
mais amplo de temas que os ODM no que diz respeito à saúde. O ODS 3, por exemplo, visa assegurar uma vida saudável e promover o bem-estar para todos, em todas as idades. Abrange os principais temas de saúde, incluindo saúde reprodutiva, materna, neonatal e infantil, doenças infecciosas, doenças crônicas não-transmissíveis, saúde mental, acidentes de trânsito, cobertura universal de saúde, saúde ambiental e fortalecimento dos sistemas de saúde. Além disso, outros ODS estão fortemente relacionados à saúde por meio das respectivas metas e indicadores, tais como nutrição (ODS 1), água e saneamento (ODS 6), qualidade do ar e violência, bem como para os principais determinantes da saúde, como a educação e a pobreza.

Figura 3. Objetivos de Desenvolvimento Sustentável (2015)
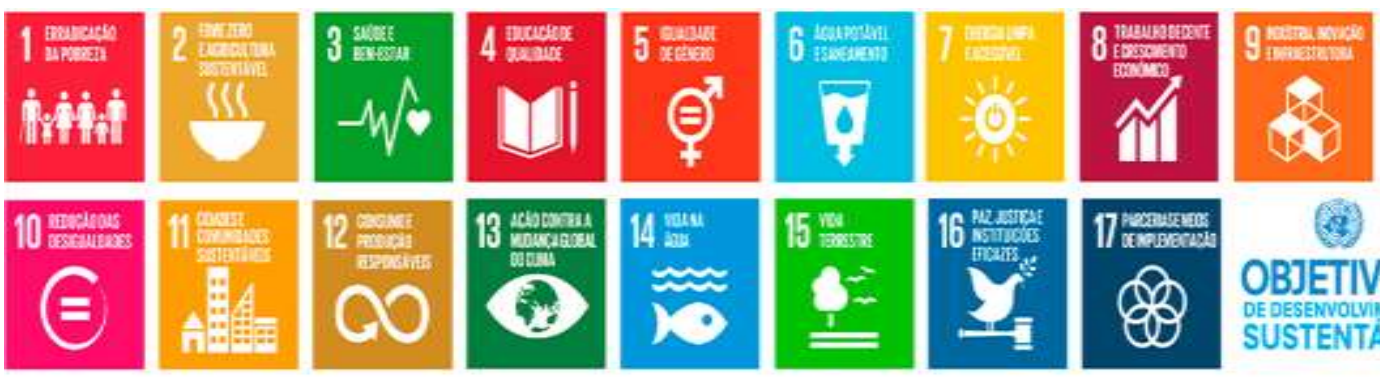

Fonte: OPAS/OMS Brasil 2020.

Como pode ser observado, os objetivos também incluem novas áreas tais como a mudança climática, desigualdade econômica, inovação, consumo sustentável, paz e justiça, entre outras prioridades, sendo interligados à chave para o sucesso de um envolverá a abordar questões mais comumente associadas ao outro. Questões ligadas à saúde podem ser observadas e impactam diretamente no trabalho contra à tuberculose.

Segundo a Organização Mundial da Saúde (2016), estima-se que um terço da população mundial esteja infectada pelo bacilo Koch e que as regiões da África e da Ásia são as que apresentam maior risco para o adoecimento.

Seis países foram responsáveis por $60 \%$ dos novos casos de tuberculose no mundo em 2015: India, Indonésia, China, Nigéria, Paquistão e África do Sul. O progresso global para o enfrentamento da doença depende de avanços na prevenção e no cuidado ao paciente em todos os países de alta carga de tuberculose, entre eles o Brasil. No mundo, o percentual de redução no coeficiente de incidência de 
tuberculose foi de $1,5 \%$ entre 2014 e 2015 . No entanto, para o alcance dos primeiros objetivos da estratégia, faz-se necessária uma redução média anual de $4 \%$ a $5 \%$ até 2020. 1 10,4 milhões de pessoas adoeceram com tuberculose em 2015. 1,8 milhão de homens, mulheres e crianças morreram de tuberculose em 2015, incluindo 400 mil pessoas vivendo com HIV. 1,1 milhão de pessoas vivendo com HIV desenvolveram tuberculose. Em 2014, 480 mil pessoas desenvolveram tuberculose multidrogarresistente, com 190 mil mortes associadas. 10,4 milhões de pessoas adoeceram com tuberculose em 2015. 1,8 milhão de homens, mulheres e crianças morreram de tuberculose em 2015, incluindo 400 mil pessoas vivendo com HIV. 1,1 milhão de pessoas vivendo com HIV desenvolveram tuberculose. Em 2014, 480 mil pessoas desenvolveram tuberculose multidrogarresistente, com 190 mil mortes associadas. (AGÊNCIA BRASIL, MS, 2017 p. 7).

De acordo com a OMS (2017), em 2015, a chamada região das Américas apresentou $3 \%$ da tuberculose no mundo, sendo computado 268 mil novos estimados, destes o Brasil com 33\%, o Peru com 14, o México com 9\% e o Haiti com $8 \%$. Foram estimados 18.500 óbitos nesta região. 7.700 casos estimados de tuberculose multidrogarresistente (TBMDR). 31.600 casos estimados de TBHIV.

A seguir, será apresentado um quadro síntese dos números de casos novos de tuberculose multidrogarresistente de 2009 a 2013 nos países: Argentina, Bolívia, Chile, Paraguai, Uruguai e Venezuela, o que nos mostra uma grande preocupação que devemos em relação aos países da América do Sul, face uma permanente circulação de pessoas entre estes países, por questões comerciais, turísticas, migratória, dentre outras.

Tabela 1. Números de casos novos de tuberculose multidrogarresistente, 20092013-Argentina

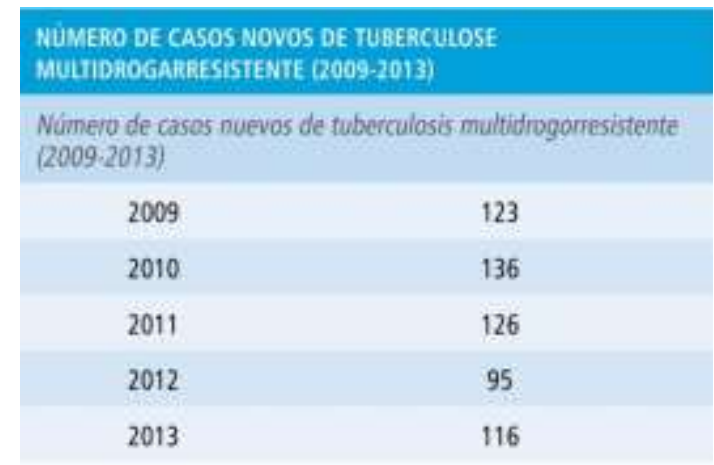

Fonte: (MINISTÉRIO DA SAÚDE, 2015) 
De acordo com o Ministério da Saúde da Argentina apud Brasil, MS (2015, p. 9), foram notificados neste país em 2013, 9.018 casos novos de tuberculose no Sistema Nacional de Vigilância em Saúde (SNVS). A taxa de incidência foi de $21,3 / 100$ mil habitantes para todas as formas de tuberculose. Do total de casos novos, 5,4\% foram coinfectados TB-HIV. Durante esse ano, foram diagnosticados 116 casos novos de tuberculose multidrogarresistente, somando 596 novos casos ao longo dos cinco anos pesquisados, (Tabela $X$ ), tendo uma taxa média anual de 119,2/ano.

Tabela 2. Número de casos novos de tuberculose multidrogarresistente, 20092014 - Bolívia

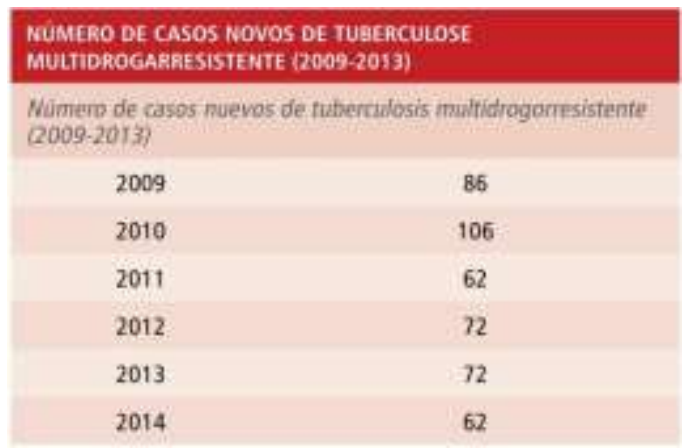

Fonte: (MINISTÉRIO DA SAÚDE, 2015)

De acordo com o Ministério da Saúde da Bolívia (2015, p. 15) em 2014, foram notificados 7.561 casos novos ao Programa Nacional de controle de Tuberculose. A taxa de incidência de tuberculose de todas as formas foi de 70,8/100 mil habitantes, e 62 casos novos de tuberculose multidrogarresistente foram diagnosticados em 2014, totalizando 460 novos casos ao longo de seis anos (Tabela X) tendo uma taxa média anual de 76,66/ano. E uma taxa média de 76,6/ano ao longo dos cinco primeiros anos da tabela. 
Tabela 3. Número de casos novos de tuberculose multidrogarresistente, 20092014 - Chile

\begin{tabular}{|c|c|}
\hline \multicolumn{2}{|c|}{$\begin{array}{l}\text { NÜMERO DE CASOS NOVOS DE TUBERCULOSE } \\
\text { MULTIDROGARRESISTENTE, } 2009-2013\end{array}$} \\
\hline \multicolumn{2}{|c|}{$\begin{array}{l}\text { Nümero de rasos nuevos de tuberculosis muftidrogorresistente, } \\
2009.2013\end{array}$} \\
\hline 2009 & 19 \\
\hline 2010 & 12 \\
\hline 2011 & 16 \\
\hline 2012 & 19 \\
\hline 2013 & 19 \\
\hline
\end{tabular}

Fonte: (MINISTÉRIO DA SAÚDE, 2015)

De acordo com o Ministério da Saúde do Chile apud Brasil, MS (2015, p.29) em 2013, foram notificados 2.190 casos novos de tuberculose de todas as formas. A taxa de incidência de 12,4/100 mil habitantes. Do total de casos deste ano $8,7 \%$ corresponde a pessoas coinfectadas com TB-HIV. Além disso, em 2013 foram diagnosticados 19 casos de tuberculose multidrogarresistente. Totalizando 85 novos casos ao longo dos cinco anos (Tabela X) tendo uma taxa média anual de 17/ano casos ao longo dos cinco anos da tabela.

Tabela 4. Números de casos novos de tuberculose multidrogarresistente, 20092014 - Paraguai

\begin{tabular}{|c|c|}
\hline \multicolumn{2}{|c|}{$\begin{array}{l}\text { NÜMERO DE CASOS NOVOS DE TUBERCULOSE } \\
\text { MULTTDROGARRESISTENTE; 2009:2013 }\end{array}$} \\
\hline \multicolumn{2}{|c|}{$\begin{array}{l}\text { Nümero de casos nuevos de tuberculosis multidrogorresistente, } \\
2009-2013\end{array}$} \\
\hline 2009 & 6 \\
\hline 2010 & 1 \\
\hline 2011 & 6 \\
\hline 2012 & 7 \\
\hline 2013 & 6 \\
\hline
\end{tabular}

Fonte: (MINISTÉRIO DA SAÚDE, 2015)

De acordo com o Ministério da Saúde do Paraguai apud Brasil, MS (2015, p. 35) em 2013, foram registrados 2.070 casos novos de tuberculose no Sistema de Informação do PNcT. A taxa de incidência de todas as formas de tuberculose foi de $34,3 / 100$ mil habitantes, em 2012. Do total de casos novos, $7,6 \%$ eram coinfectados com TB-HIV. Em 2013, foram diagnosticados seis casos novos de tuberculose multidrogarresistente. Totalizando 26 novos casos ao longo dos 
cinco anos (Tabela $X$ ) tendo uma taxa média anual de 5,2/ano casos ao longo dos cinco anos da tabela.

Tabela 5. Números de casos novos de tuberculose multidrogarresistente, 20092014 - Uruguai

NÚMERO DE CASOS NOVOS DE TUBERCULOSE
MULTIDROGARRESISTENTE, 2009-2013
\begin{tabular}{ccc|} 
Número de casos nuevos de tuberculosis multidrogorresistente, \\
$2009-2013$ \\
Ano/Año & Testados/Testados \% & MDR/MDR \% \\
2011 & 80,7 & 0,2 \\
2012 & 85,7 & 0 \\
2013 & 71,9 & 0
\end{tabular}

Fonte: (MINISTÉRIO DA SAÚDE, 2015)

Em 2013, foram registrados 808 casos novos de tuberculose e 70 de retratamento no registro Nacional de Tuberculose do PNT. A taxa de incidência para todas as formas foi de 25,9/100 mil habitantes. Do total de casos novos, 107 (13,2\%) apresentavam coinfecção TB-HIV. Em 2013 foi registrado um caso de tuberculose multidrogarresistente (TB-MDr) correspondente a um paciente previamente tratado, e nenhum com tuberculose extensivamente resistente (TBXDr).

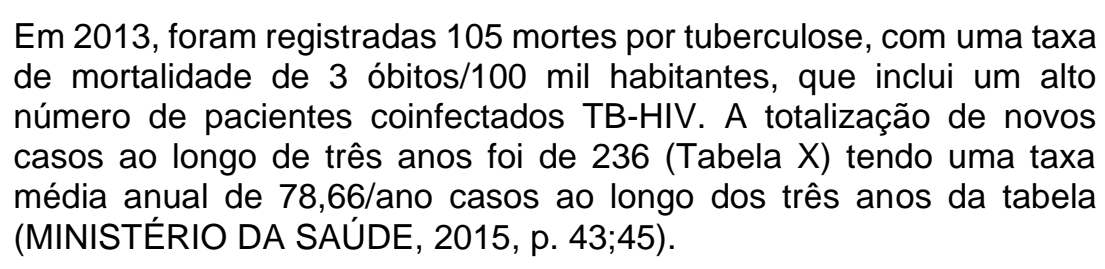

Tabela 6. Números de casos novos de tuberculose multidrogarresistente, 20092014 - Venezuela

\begin{tabular}{|c|c|}
\hline \multicolumn{2}{|c|}{$\begin{array}{l}\text { NUMERO DE CASOS NOVOS DE TUBERCULOSE } \\
\text { MULTIDROGARRESISTENTE, 2009-2013. }\end{array}$} \\
\hline \multicolumn{2}{|c|}{$\begin{array}{l}\text { Numero de casos nuevos de tuberculosis multidrogorresistente, } \\
2009-2013\end{array}$} \\
\hline 2009 & 21 \\
\hline 2010 & 21 \\
\hline 2011 & 25 \\
\hline 2012 & 21 \\
\hline 2013 & 19 \\
\hline
\end{tabular}


Para 2013, a taxa de incidência foi de 20,8/100 mil habitantes, o que representa uma redução de $0,3 \%$ em relação ao ano anterior. Em relação à coinfecção TB-HIV, o número de casos aumentou de maneira estável desde 1990; testagem para HIV foi realizada em $70 \%$ dos casos notificados e o percentual de coinfecção, em 2013, foi de $7,6 \%$ nos casos novos. Nesse ano foram diagnosticados 19 casos de tuberculose multidrogarresistente, com uma média de 21 casos nos últimos cinco anos. Ministério da Saúde do Venezuela apud Brasil, MS (2015, p. 49).

Assim, pode-se observar que a questão da tuberculose tanto no Brasil quanto no mundo ainda é motivo de preocupação de todos. Espera-se que este estudo seja o ponto de partida para que a temática seja aprofundada e divulgada como imperiosa na comunidade médica e entre a população como um todo.

\section{METODOLOGIA}

Esta pesquisa é caracterizada por uma revisão bibliográfica de natureza qualitativa. A coleta de dados foi realizada na plataforma Google Acadêmico no período de janeiro a outubro de 2020 em trabalhos somente em português com as palavras-chave "Tuberculose"; "Prevenção" e "Saúde".

\section{CONSIDERAÇÕES FINAIS}

Observa-se que há um esforço contínuo da comunidade médica e de apoio à saúde para a prevenção, por meio de constantes campanhas vacinais, e para o correto e completo tratamento para a tuberculose, que costuma ser longo e demanda certa paciência para os pacientes acometidos.

Em paralelo, observa-se que junto à tuberculose tem-se o HIV que fragiliza o paciente, tornando-o mais suscetível ao contágio e, ainda, dificultando a cura efetiva da doença.

O desconhecimento da população mundial somado a algumas nações que não orientam a população para a vacinação com a vacina BCG nos primeiros dias de vida, agravam o problema. Campanhas antivacinistas parecem ter ganhado força nos últimos anos, trazendo prejuízos diversos para a população de modo geral e gerando mais letalidade para doenças que antes já haviam sido controladas. 
Aprofundar conhecimentos, portanto, sobre uma doença que tem vacina e cura, entretanto ainda é causadora de tantas mortes na atualidade, é imperioso e necessário para a manutenção da vida humana.

\section{REFERÊNCIAS BIBLIOGRÁFICAS}

AGÊNCIA BRASIL. Tuberculose matou 1,8 milhão de pessoas em 2015, diz OMS. Disponível em: https://agenciabrasil.ebc.com.br/geral/noticia/201610/tuberculose-matou-18-milhao-de-pessoas-em-2015-diz-oms. Acesso em 15 out. 2020.

BRASIL. Ministério da Saúde. Secretaria de Vigilância em Saúde. Departamento de Vigilância das Doenças Transmissíveis. Brasil Livre da Tuberculose: Plano Nacional pelo Fim da Tuberculose como Problema de Saúde Pública / Ministério da Saúde, Secretaria de Vigilância em Saúde, Departamento de Vigilância das Doenças Transmissíveis. - Brasília: Ministério da Saúde, 2017. $52 \mathrm{p}$.

CORREIO BRAZILIENSE. Cientistas da Suíça descobrem que tuberculose surgiu na África. Disponível em:

https://www.correiobraziliense.com.br/app/noticia/ciencia-esaude/2013/09/15/interna ciencia saude,388177/cientistas-da-suicadescobrem-que-tuberculose-surgiu-na-africa.shtml Acesso em 15 out. 2020.

DUARTE, R. Artigo de Revisão: Teste Tuberculínico. Como optimizar? Rev Port Pneumol, Vol XV (2): 295-3 04. Março/Abril. 2009.

OPAS BRASIL. 10 principais causas de morte no mundo. Disponível em: https://www.paho.org/bra/index.php?option=com content\&view=article\&id=563 8:10-principais-causas-de-morte-no-mundo\&ltemid=0 Acesso em 30 de março de 2020. 
OPAS BRASIL. Objetivos de Desenvolvimento Sustentável. Disponível em: http://www.itamaraty.gov.br/pt-BR/politica-externa/desenvolvimentosustentavel-e-meio-ambiente/134-objetivos-de-desenvolvimento-sustentavelods Acesso em 30 de março de 2020.

MINISTÉRIO DA SAÚDE. Situação Epidemiológica da Tuberculose nos Estados Partes e Associados do MERCOSUL 2009 a 2013. Brasília - DF, 2015.

Disponível em: http://www.riocomsaude.rj.gov.br/Publico/MostrarArquivo.aspx?C=Qlc8jVAKFF Q\%3D. Acesso em 15 out. 2020.

MINISTÉRIO DA SAÚDE. Gabinete do Ministro. Secretaria de Vigilância em Saúde. Situação epidemiológica da tuberculose nos estados parte e associados do Mercosul 2009 a 2013 = Situación Epidemiológica de la Tuberculosis en los Estados Parte y Asociados del Mercosur 2009 a 2013. Editora MS. 2015. http://www.riocomsaude.ri.gov.br/Publico/MostrarArquivo.aspx?C=Qlc8jVAKFF Q\%3D acesso em 05 de março de 2020.

PERDIGÃO, Paula. Manual clínico de tuberculose. Apoio aos Cursos Clínicos Moçambique. 2008. https://www.who.int/hiv/pub/guidelines/mozambique tb.pdf acesso em 10 de março de 2020.

SANTOS, Vanessa dos. 1o de julho - Dia da Vacina BCG. Disponível em: https://brasilescola.uol.com.br/datas-comemorativas/dia-da-vacina-bcg.htm. Acesso em 15 out. 2020.

TEIXEIRA, Gilmário M. Boletim Pneumologia Sanitária v.11 n.2 Rio de Janeiro dez. 2003 